\title{
Video Content Delivery Schemes: Approaches and Directions
}

\author{
Diego Pajuelo \\ Yuzo Iano \\ David Minango \\ Gabriel Gomes de Oliveira \\ Ana Carolina Borges \\ Reinaldo Padilha
}

\section{CITE THIS ARTICLE}

Pajuelo, Diego, lano, Yuzo, Minango, David, de Oliveira, Gabriel Gomes, Borges, Ana Carolina, Padilha, Reinaldo; 2019. Video Content Delivery Schemes: Approaches and Directions. SET INTERNATIONAL JOURNAL OF BROADCAST ENGINEERING. ISSN Print: 2446-9246 ISSN Online: 2446-9432. doi: 10.18580/setijbe.2019.10. Web Link: http://dx.doi.org/10.18580/setijbe.2019.10 


\title{
Video Content Delivery Schemes: Approaches and Directions
}

\author{
Diego Pajuelo, Yuzo Iano, Member, IEEE, David Minango, Gabriel Gomes de Oliveira, Ana Carolina Borges, \\ Reinaldo Padilha
}

\begin{abstract}
This paper addresses the main two types of video delivery networks deployed into digital television services throughout the years. Underlying and Overlay networks are described in order to introduce how the physical infrastructure and network architecture impacted on the modeling of the latest video content delivery schemes. The convergence of different video delivery networks, in a technical sense, is a real thing and may disrupt the broadcasting industry in the long-term.
\end{abstract}

Index Terms-Broadcasting, IPTV, OTT, video delivery network.

\section{INTRODUCTION}

D URING the last decade, the explosion of access to videocontent over the current networks by users all-over-theworld has been remarkable. As more people are experiencing better video-based services, called as killer-applications, daily Internet traffic has increased considerably from the ISP (Internet Service Provider) edge, in some cases more than doubled regarding the evolution of five years [1]. As a consequence, new video content delivery networks have been appeared, driven by the erosion of the heterogeneous networks and devices, for instance, adaptive video streaming scheme over wireless networks [2] or global overlay live streaming [3] proposals are crossing the same network domains, seeking to optimize the end-to-end video content delivery and reach the best QoE (Quality-of-Experience) of end-users.

On the other hand, one important factor to consider that really arouses this audiovisual content tsunami over the Internet is the great efficiency that the video encoder has achieved in recent years. It is known that a new technological evolution on video codecs is when compression ratio reaches $50 \%$ with respect to its predecessor codec. Benjamin Bross, who is with Germany's Fraunhofer Henrich Herts Institutes, states that: "the more efficient we are in compressing video, the [easier] and more accessible it is for people to watch HD (High Definition) video at home and so more and more people watch HD video at home "[4], the so-called 'Jevons Paradox'. This remarkable codec efficiency without precedent in the audiovisual world allows to offer better content and attract a new audience, hungry to see innovator productions of high artistic quality. Telecommunication engineers must be aware of these changes and global trends to be able to propose efficient end-to-end video content delivery schemes, either re-modeling the current layering architecture based on cross-layer design or deploying new physical network.

Video codec efficiency has undoubtedly been achieved in multimedia industry, but the video content is really the thing more meaningful by the users against the transmission technical details. Citing to Matthew Postgate, CTO of the BBC, says "People interact with TV in search of content that contains interesting stories and engages them"[5]. By many time, big players in television networks deployed means of communications based on different technologies, as were Cable TV (Television), Satellite TV (Sat-TV) and Over-theAir TV (OTA), to deliver video content. Something in common was the type of delivery, broadcasting or point-to-multipoint transmission scheme. In recent years, with the emergence of the so-called Over-the-Top (OTT) services, delivery of pointto-point transmissions schemes for video content allowed to the arrival of productions with high quality in the mainstream media, paving the way to a revolutionary video streaming service. The notion on which it is supported is that it can reach a global relevance with relative less infrastructure using the Internet as its main technological partner. These services are become a new concept of watching television, it drastically changed the behavior of users and viewing experience, giving greater comfort and more interactivity. This phenomenon did impact the audiovisual industry, leading to the branded TV companies has come into this ecosystem, where the content is the king of the jungle. People are no longer move between channels, instead, people tend to change of content, which is in turn, in a technical sense, going to another physical infrastructure or network architecture.

The domains involved in this audiovisual ecosystem try to decipher the path to follow in a disruptive technological environment. This paper explores both technological and academic approaches, compiling the main video delivery networks deployed in the latest years, the schemes adopted to face with communication channel impairments; and by last, to analyze how those schemes drive the future networks and which are the directions that they are aiming at, where growing diversity of TV content is a consequence of this fast-changing digital era.

The remaining of this paper is organized as follows: Section II presents a technical overview of Video Delivery Networks. Section III discusses about the latest Video Content Delivery Schemes. Finally, Section IV reveals the final remarks and trends.

\section{VIDEO DELIVERY NETWORKS}

Since the releasing of Television as a community service, Video Delivery Networks were modeled about the notion of a point-to-multipoint transmission scheme over an underlay network, After that, new television services came to light that offered more variety of programs; Cable TV was the pioneer 
service to distribute television channels over coax, afterwards Satellite TV was inserted in this industry thanks to the rapid development of satellite communications that allow to land up at the households. The Over-The-Top services came out in the last years and it seems to be a promissory technology. This whole environment configures the TV entertainment industry just as it is. In this section, two concepts will be discussed, Overlay and Underlay Networks, in order to highlight the importance of their main ideas and how can contribute to the modeling of video delivery networks.

\section{A. Underlay Networks}

The Underlay Network is physical infrastructure in telecommunications, concerning all physical elements and interfaces comprehended in a communication system, either simplex, half-duplex or full-duplex; whose main feature is the establishment of a dedicated communication channel between interested parties. In particular, the deployment of infrastructure was mandatory for broadcasters because there were no networks spread across an entire region or worldwide back in the $50 \mathrm{~s}$, for instance, the Internet originated in the late 1960s with a world footprint in the mid-90s. Television networks can be classified as: private and public networks. Cable TV and Satellite TV providers make use of walledgarden environments, but Over-the-Air TV uses the shared radio frequency spectrum under the concept of 'free-to-air' transmission instead.

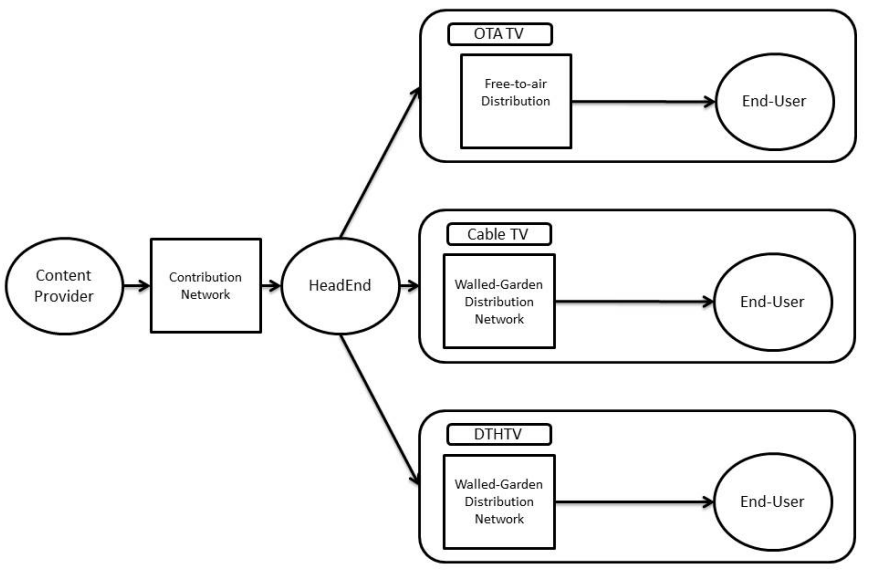

Fig. 1. Broadcast Architecture for TV

As shown in Figure 1, the Contribution Network and Headend are common for these three types of commercial television platforms. The Contribution Network is in charge of delivery video content from different content providers; producing live feeds, transmitting local video sources, gathering Video-On-Demand (VOD) Content or video content from the Internet; to Headend. In the Headend, all television signals are captured, processed and modulated in digital channels before distribution.

About the distribution network, technologies on Cable TV first delivered digital video signals were implemented using
HFC (Hybrid-Fiber-Coax) technology and lately that one was surpassed by the FTTh (Fiber-To-The-home) technology. Moreover, Direct-To-Home Television (DTHTV) delivers digital video signals through a direct broadcast satellite provider. To be specific, Eutelsat, one of the word's leading satellite operators, "reaches over 274 million homes across Europe, Middle East, and Africa at 20 key video neighborhoods"[6]. The analog-digital transition implied to broadcasters, from a technical perspective, delivering more television channels with diversified programming for end-users over distribution networks, leveraging so, the infrastructure already built by themselves.

Free-to-air distribution or terrestrial broadcasting does not contemplate physical intermediary nodes or network equipment but sends television signals via line-of-sight radio propagation until the end-user. Its main technologies have taken the lead when it comes to innovations in digital processing techniques for communication as opposed to walled-garden distribution technologies because they had to face with time and frequency selective fading channels, whose modeling remains a challenge to this day. These technological advances focused on optimizing physical layer performance by means of supporting a wide range of transmission parameters in order to satisfy the optimal trade-off between robustness and throughput according to broadcasters decisions. In this respect, Table I details the different transmission parameters of the most advanced terrestrial broadcasting standards; ISDB-T/Tb [7], DVB-T2 [8] and ATSC3.0 [9]. It is a fact that OFDM (Orthogonal Frequency Division Multiplexing) modulation is the cornerstone for television waveform generation and is a general consensus. Figure 2 shows the signal processing diagram of the protection-at-the-bit-level stage and the constellation mapper module, which fulfills the function of improving spectral efficiency; ISDB-T/Tb standard named it as the modulation and error protection module and DVBT2 and ATSC3.0 standards as Bit Interleaved Coding and Modulation (BICM) module, it is important to mention that Outer Encoder evolved from Reed Salomon codes to BCH (Bose, Ray-Chaudhuri and Hocquenghem) codes and Inner encoders evolved from Convolutional codes to LPDC (LowDensity Parity Check) codes, both forms part of the Forward Error Correction (FEC) codes. According to this work [10], the performance of the ATSC 3.0 BICM reaches very closely to the upper bound capacity, in terms of spectral efficiency, proposed by Shannon in AWGN (Additive white Gaussian noise) channel.

Before framing, ATSC 3.0 incorporates a constellation superposition technology unlike DVB-T2 and ISDB-T/Tb standards, known as LDM (Layered Division Multiplexing). It allows to multiplex two data streams; the BICM Core Layer (CL) and the BICM Enhanced Layer (EL); at different power levels [11]. In practical systems, the Core Layer delivers robust mobile broadcasting services, it means that operates with negative CNR (Carrier-to-Noise ratio), and the Enhanced Layer delivers UHDTV (Ultra-High Definition Television) or multiple HDTV (High Definition Television) services. After LDM processing, Time Interleaving (TI) module is designed for coping with long burst errors in the time domain [12]. A 

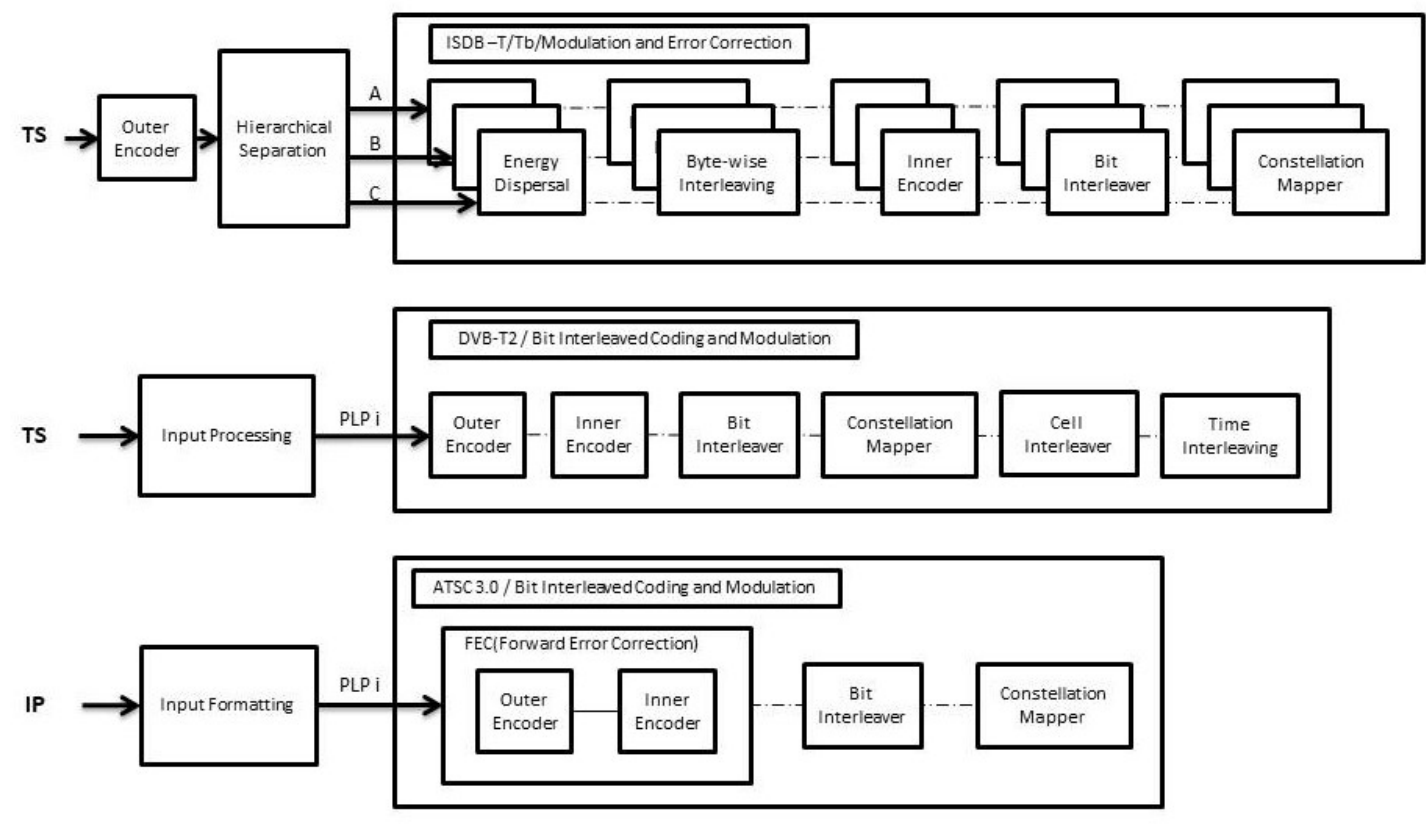

Fig. 2. Protection-at-the-bit-level and Modulation Stage,adapted from [7], [8] and [9]

salient difference is at the moment of the Framing process, ISDB-T/Tb applies Frequency Interleaver (FI) after the TI process, but DVB-T2 and ATSC3.0 wait for Framing process first and after FI is applied. As the same of TI, Frequency Interleaving (TI) module is designed for separating bursts errors in frequency domain. As the last stage, the pilot carriers, that are used for channel estimation, are inserted into the final OFDM symbol and the Guard Interval (GI) is time-aligned with the OFDM symbol in order to mitigate ISI (Inter-Symbol Interference). Figure 3 shows the entire process of the OFDM waveform generation.

One of the contributions that have been maintained over time was the sending of signaling information. ISDB-T/Tb introduced the TMCC (Transmission Multiplexing Configuration Control) signal providing to a broadcaster the option to configure different transmission schemes that are tailored to servicespecific robustness levels [7]. Basically, an arrange of carriers of an OFDM segment were set up to deliver the transmission parameters (inner-code, coding rate, modulation scheme, and time interleave) of each layer, up to three hierarchical layers (A, B or C). Also, this scheme was very useful for narrowband mobile receivers. Following this thread, L1 signaling information, in DVB-T2 and ATSC3.0, carrying information about how Physical Layer Pipes (PLPs) are allocated in the frame and their respective physical parameters, as well as the waveform parameters of the Preamble OFDM symbols [8], [9]. This flexibility at the transmission level was extrapolated to the link layer, ATSC 3.0 encapsulated the binary input streams into ALP (ATSC Link-Layer Protocol) packets for letting generic data streams as a valid input source to the system, for instance, the IP packets or Transport Stream could be part of the same channel [9].

It is clear that the motto driving the terrestrial broadcasting is focused on strengthening the receiver signal for rooftop antenna (fixed) and mobile receivers, however, backward compatibility has been always the main drawback of worldwide television standards. For this reason, some kind of information about incoming signals are mandatory, DVB T2 launched a first proposal inserting the P1 symbols within the T2 frame. This enables synchronization and signaling of the entire frame but does not resolve the problem [8]. Based on these ideas, ATSC 3.0 disclosed the bootstrap symbol signaling the most basic information, such as the version of the ATSC 3.0 standard, the Emergency Alert Service (EAS) wake up bit, the sampling rate of the current frame and the preamble structure [9]. Really, this technology could disrupt the telecommunication industry, enabling cooperative transmission between different network operators [13].

This sub-section summarizes the physical layer architecture of terrestrial broadcasting and the evidence proves that broadcasters invested in improving point-to-multipoint transmission without involving the redesign of network architecture by leveraging all the resources of a dedicated communication channel. However, streaming video services are increasingly popular over the Internet and competing with the mainframe television infrastructure. By not owning one, these services are being born on the concept of an overlay network, that will be detailed in the next sub-section.

\section{B. Overlay Networks}

The Overlay Network refers to the approach of adding resources on top of an existing network infrastructure [15].These resources not only cover services such as a local proxy, but services that can be offered anywhere in the world without having a network deployed directly to the client. Internet could be the case study to explain the main ideas about overlay network. Despite its network infrastructure was never designed to be optimal for any particular application [16], in the case 


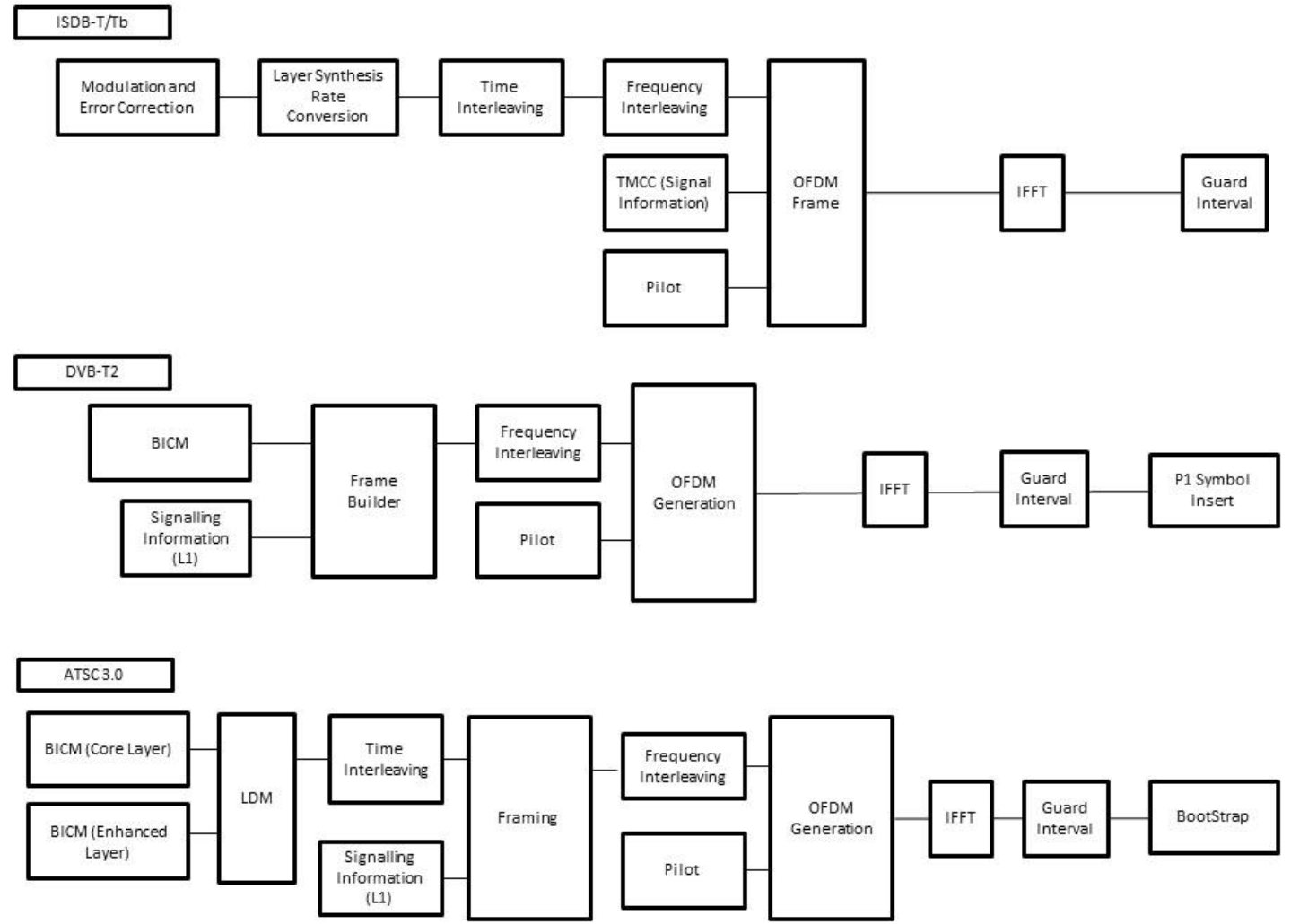

Fig. 3. OFDM Waveform Generation, adapted from [7], [8] and [9]

of the access network or 'last mile' in Internet Infrastructure, the PSTN (Public Switched Telephone Network) network and the walled-garden TV networks, or others underlay networks, enable the access from end-users to the Internet. The ADSL (Asymmetric Digital Subscriber Line) technology was spread over the PSTN network, the DOCSIS (Data Over Cable Service Interface Specification) 3.0 protocol [17] did the same thing in a HFC network, as well as the GPON (Gigabit-capable Passive Optical Network) [18] in FTTh network. Hence, it was no suprise that local area networks, such as the IEEE 802.11 Standards Body [19], and the latest cellular generation released in 2011, the LTE Advanced [20], made possible the final convergence of all networks over IP-based technologies, known as broadband networks.

The operators in their constant search to improve their services decided to invest on deployment Internet network backhaul within their own core network as a manner of adding new applications over their last-mile connection. The MNOs (Mobile Network Operator) saw this opportunity to build a infra-based overlay network. Hence, experiences such as the EPC (Evolved Packet Core) network serves to the MNOs as, on the one hand, a management system between end-user and MNO, called as Control Plane, and, by the other hand, as a virtual interface at the application level, whether for external connections, or a local mobility anchor for MNO's internal purpose [21]. Figure 4 depicts the EPC arquitecture, the Radio Acces Network (RAN) involves the eNodeB (Enhanced Node
B) and the User Equipment (UE) of end-user. The MME (Mobility Management Entity) is in charge of all the control plane functions, e.g. the end-user authentication process works with the Home Subscriber Server (HSS), the management of end-user QoS and the tracking update process. Moreover, the GW (Serving Gateway) receives the incoming packets and routing them to the eNodeB, whose end-user currently stays after the respective displacement in a mobility context. The closest entity to the Internet is the Packet Data Network Gateway (PDN-GW) and its function is similarly as Layer2/3 router in the backbone Internet [21]. In recent years, MNOs operators are pushing the development of IP Multimedia Subsystem (IMS) because can provide the multimedia services over IP in next-generation mobile networks with the intention of surpassing the current OTT services by offering slices of network resources inside its infrastructure in order to guarantee a high QoS for real-time applications [22].

The same problem was experienced in the television industry. As were explained in the previous sub-section, television networks distributed digital video signals in broadcasting mode restricted to the radio frequency bands of the physical transmission medium. In fact, with an ever-improving video codec efficiency, more channel programs were multiplexed over a single RF thanks to MP2T (MPEG-2 Transport Stream) packets [23]. Nevertheless, this increasing programming number of options did little to impact the viewing behaviors[24]. For instance, cable channels rose by more than $45 \%$ between 
TABLE I

Comparison Overview Between Terrestrial Digital Television Standards In 6Mhz, Adapted From [9], [14] ANd [7]

\begin{tabular}{|c|c|c|c|}
\hline Parameter & ISDB-T (1997) / ISDB-Tb (2006) & DVB-T2 (2008) & ATSC 3.0 (2016) \\
\hline $\begin{array}{c}\text { Input } \\
\text { Formatting }\end{array}$ & Hierarchical Transmission $(\mathrm{A}, \mathrm{B}, \mathrm{C})$ & PLP & PLP \\
\hline Outer Code & Reed-Salomon code & $\mathrm{BCH}$ & $\mathrm{BCH}, \mathrm{CRC}$, none \\
\hline Inner Code & Convolutional code $(1 / 2,2 / 3,3 / 4,5 / 6,7 / 8)$ & LPDC code $(1 / 2,3 / 5,2 / 3,3 / 4,4 / 5,5 / 6)$ & $\begin{array}{c}\text { LDPC code }\left\{\begin{array}{c}2,3,4,5,6,7,8,9,10,11 \\
12,13\} / 15\end{array}\right.\end{array}$ \\
\hline $\begin{array}{l}\text { Constellation } \\
\text { Mapper }\end{array}$ & QPSK, 16-QAM, 64-QAM & QPSK, 16-QAM, 64-QAM, 256-QAM & $\begin{array}{c}\text { QPSK, 2D-16NUC, 2D-64NUC, } \\
\text { 2D-256NUC, 1D-1024NUC, 1D-4098NUC }\end{array}$ \\
\hline Multiplexing & FDM & TDM & TDM legacy/TDM-FDM hybrid/LDM \\
\hline Modulation & Band Segmented Coded OFDM & Coded - OFDM & OFDM \\
\hline FFT size & $2 \mathrm{~K}, 4 \mathrm{~K}$ and $8 \mathrm{~K}$ & $1 \mathrm{~K}, 2 \mathrm{~K}, 4 \mathrm{~K}, 8 \mathrm{~K}, 16 \mathrm{~K}$ and $32 \mathrm{~K}$ & $8 \mathrm{~K}, 16 \mathrm{~K}$ and $32 \mathrm{~K}$ \\
\hline $\begin{array}{l}\text { GI (Guard } \\
\text { Interval) }\end{array}$ & $1 / 4,1 / 8,1 / 16,1 / 32$ & $1 / 128,1 / 32,1 / 16,19 / 256,1 / 8,19 / 128,1 / 4$ & $\begin{array}{c}3 / 512,3 / 256,1 / 64,3 / 128,1 / 32,3 / 64,1 / 16, \\
19 / 256,3 / 32,,\{57,512,3\} / 16,1 / 8, \\
19 / 128,1 / 4 \text { (symbol and time-aligned } \\
\text { frames) }\end{array}$ \\
\hline $\begin{array}{l}\text { Multiple } \\
\text { PLPs per } \\
\text { service }\end{array}$ & - & No (1 commom PLP) & Yes (up to 4) \\
\hline Link layer & MPEG2-TS & MPEG2-TS & ALP (ATSC Link-Layer Protoco) \\
\hline $\begin{array}{c}\text { Main } \\
\text { Transport } \\
\text { Protocol }\end{array}$ & TS & TS & IP \\
\hline $\begin{array}{l}\text { Min-Max } \\
\text { Data Rate } \\
6 \mathrm{Mhz}\end{array}$ & 3,65 Mbps - 23,23 Mbps & 5,6 Mbps - $38 \mathrm{Mbps}$ & $1 \mathrm{Mbps}-57 \mathrm{Mbps}$ \\
\hline $\begin{array}{l}\text { Channel } \\
\text { Bonding }\end{array}$ & - & No & Yes (Two RFs) \\
\hline $\begin{array}{l}\text { Video } \\
\text { Codec } \\
\text { Support }\end{array}$ & H.264/AVC & H.264/AVC & H.265/HEVC \\
\hline $\begin{array}{l}\text { SNR } \\
\text { operating } \\
\text { range } \\
\text { AWGN }\end{array}$ & $3,2 \mathrm{~dB}-19,8 \mathrm{~dB}$ & $+1 \mathrm{~dB}, 22 \mathrm{~dB}$ & $-6,2 \mathrm{~dB},+32 \mathrm{~dB}$ \\
\hline
\end{tabular}

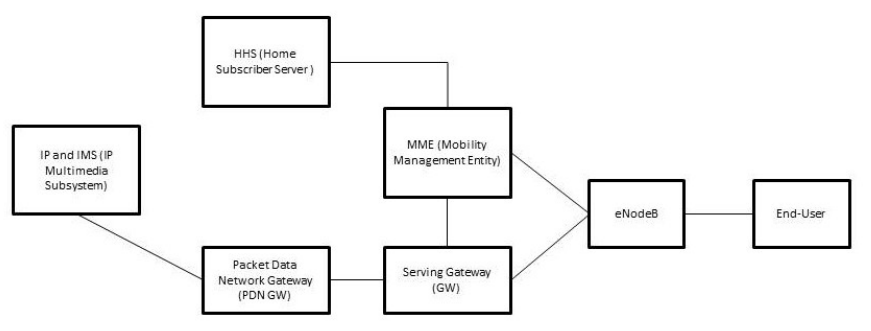

Fig. 4. The Evolved Packet Core Arquitecture, adapted from [21]

2008 and 2013 in the North-American industry reaching a total amount of 180 channels, but users continued to consume from an average of 17 or 18 channels [25]. In that sense, the inefficient use of resources within the broadcasting gave rise to the appearance of new multimedia platforms such as the media overlay networks, whose leading technology was the IPTV.

The IPTV (Internet Protocol Television) platform was the first effort to take advantage of the resources of enterprise private networks in order to provide a more personalized service to the user by leveraging the whole IP stack protocols and the full-duplex communication. Hence, additionally to provide live channels and Video on demand (VOD), new types of services were offered to final users, such as Schedule TV services, Content on demand (COD), Personal Video
Recorder (PVR), Information service, Communication service, Notification Service and Advertisement service [26], so to improve the audiovisual interactivity and addressability.

Legacy IPTV was deployed into managed operator networks over the traditional broadcasting system for the sake of competing with the conventional cable and satellite TV services in terms of $Q o S$, full-HD transmission with above $6 \mathrm{Mbps}$ video rate and a transport delay shorter than 2 seconds [27] by aggregating layer 3 capabilities, by redesigning network topology and by offering to the users web services in order to make more efficient video content delivery and render a more interactive product. By contrast, just a few ISPs were prepared to modernize their underlying networks and be became as IPTV providers, because of the large initial investment and a high operational cost. Hence, only network operators that owned a large metropolitan area infrastructure can be able to commercialize varied IPTV packages until the emergence of OTT service providers.

Broadband networks have been experiencing the gradual growth of data access speeds which caused that the constraint on bandwidth when delivering video content over these networks be lifted, either local or mobile networks. This technological breakthrough opened countless opportunities for content creators and video-service providers. The emergence of $O T T$ services over unmanaged networks consolidated an agile and flexible communication system, being modeled to interact with the user giving him the opportunity to have 
control of the content he wants to see at anywhere and anytime, which was rarely possible with traditional broadcasting or IPTV systems. For instance, user-generated content applications such as YouTube is watched by 5 billion people per day [28] and everything indicates that it is expanding thanks to the proliferation of Content Delivery Networks (CDN) within the large Internet infrastructure [29].

This section summarizes the two kinds of networks deployed in the television industry, highlighting their main innovations and drawbacks. New video delivery schemes are been addressed in the last years driven by technological innovations and new user demands.

\section{Latest Video Content Delivery Schemes}

So far, the networks that participate in the process of delivering video content to the final user have been discussed above. An efficient end-to-end video content delivery scheme should consider upon which type of network is being deployed, e.g. which network components are reachable and manageable by the provider, since this will define the type of technology to use and the business model to be implemented. For this reason, the latest video content delivery proposals model enhanced video transmission systems based on cutting-edge technologies, leveraging novel mathematical approaches and heuristic methods which seek to find the most optimized video content delivery scheme over resource-constrained networks.

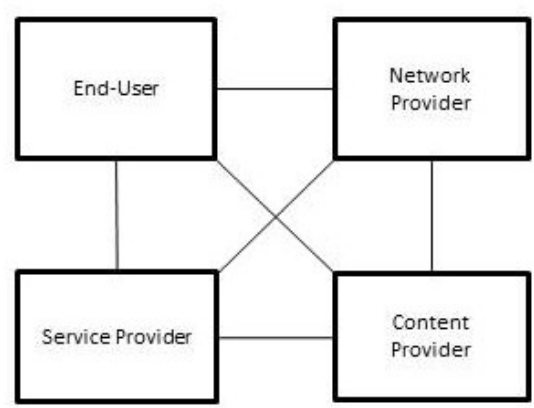

Fig. 5. IPTV domains, adapted from [30]

Fig 5 depicts the four domains of IPTV service provisioning [30]:

1) Content Provider: who owns the content copyright for distribution.

2) Service Provider: Generally is who offers a telecommunication service, but recently CDN's owner performs the same work for content providers.

3) Network Provider: who really reaches and manages the network equipment and is the first hop node form enduser edge.

4) End-User: who enjoys the service.

In contrast to the architecture of Fig 1, The IPTV architecture allows direct interaction of the content provider with the end-user, without having to go necessarily through a service provider. From this moment, new video-service providers emerged and disrupted the television industry in an unexpected way. Netflix's service, the most popular streaming video service, takes advantage of the multiple cloud technologies that exist to offer a value-added video service to end-users. Netflix employs three popular CDNs with footprint worldwide to serve its subscribers through chunked-based delivery, enabling adaptive video streaming to face unstable and unreliable channels [31]. In 2018, Netflix's traffic is responsible for $26.58 \%$ of global video streaming traffic share according to [32].

Commonly, the television protocols focus on strengthening the signal-to-noise ratio and increasing the spectral efficiency of the physical transmission medium, but the sudden appearance of streaming services over packet-based networks made the audiovisual industry face new challenges. Two types of schemes are been discussed in the latest years: push-based schemes (server pushes the to client such as broadcastingbased technologies) and pull-based scheme (client pulls video content from the server). Real-Time Streaming Protocol (RTSP)/Real-time Transport Protocol (RTP) fits well in the first scheme and relies on just-in-time data delivery with just-in-time rendering [33] and HyperText Transfer Protocol (HTTP) streaming is a pull-based scheme, very popular for file delivery, that achieves near-live streaming due to the user needs to wait until the buffer is ready to render the first Group of Pictures (GOP) affecting the end-to-end delay. This fact notwithstanding, there is a subclass of HTTP-based streaming, known as adaptive multimedia streaming schemes, It is mainly modeled to adapt to the instabilities of the transmission channel such as losses, delay and time-varying bandwidth, typical of wireless transmission and can be classified as: transcodingbased, scalable video coding, multi-layer encoding, multiple description coding and independent bitrate encoding solutions [33]. Hence, hybrid schemes that make use of the best of both proposals have become visible, deploying in favored video-service applications, for instance, Periscope allows users broadcasting over the Internet. To achieve this, the first 100 viewers access to the real-time server for minimizing the end-to-end latency and the rest of them pushes the video content from the adaptive streaming media server for reducing processing overhead [34].

It is worth mentioning that the advent of CDN networks inside the Internet allowed new distribution and transmission schemes in the different layers of the Open Systems Interconnection (OSI) stack. It is an environment where new OTT stakeholders, which involves Content Providers, CDNs and ISPs, want to position on the live TV broadcast market. Akamai, the worlds largest and most trusted cloud delivery platform, "forecasts that 500 million viewers will soon be watching prime-time live sports online" [26]. According experimental tests, low-Latency chunked streaming schemes enables ranged from 2.3 to 3 seconds of delay with acceptable QoE [35]. Moreover Layer 3 capabilities and beyond were necessary to make viable this type of architectures. IP multicast, Peer-to-peer (P2P) Sharing and Proactive caching [26] are the most promising systems in order to alleviate unicast limitations. 


\section{FINAL REMARKS AND TRENDS}

Future video content delivery networks will be modeled as a media overlay network with global coverage with IP convergence and ubiquitous radio access through distributed array antennas. However, it still too soon to forecast how will be the market model that allows a free-competition scenario and be able to incentive the emergence of new video producers with better content to offer to the end-user.

\section{ACKNOWLEDGMENT}

The authors would like to thank the CAPES (Coordenao de Aperfeioamento de Pessoal de Nvel Superior) and FAEPEX (Fundo de Apoio ao Ensino, Pesquisa e Extenso) programs for the financial support and the academic incentive.

\section{REFERENCES}

[1] M. Trevisan, D. Giordano, I. Drago, M. Mellia, and M. Munafo, "Five years at the edge: Watching internet from the ISP network," CoNEXT 2018 - Proceedings of the 14th International Conference on Emerging Networking EXperiments and Technologies, pp. 1-12, 2018.

[2] J. Guo, X. Gong, J. Liang, W. Wang, and X. Que, "An Optimized Hybrid Unicast/Multicast Adaptive Video Streaming Scheme over MBMSEnabled Wireless Networks," IEEE Transactions on Broadcasting, vol. 64, no. 4, pp. 791-802, 2018.

[3] D. Ren, Y. Xu, and S. H. Gary Chan, "Beyond 1Mbps global overlay live streaming: The case of proxy helpers," ACM Transactions on Multimedia Computing, Communications and Applications, vol. 11, no. 2, 2014.

[4] J. E. O'Neal, "BMSB Attracts Video/Broadband Experts From Around The World," IEEE Broadcast Technology, vol. 3, p. 8, 2018.

[5] D. F. Feitosa, "Transmissão híbrida na TV aberta brasileira: o DTV Play e as suas potencialidades," Revista da SET, jan 2019. [Online]. Available: http://www.set.org.br/news-revista-da-set/revista/transmissaohibrida-na-tv-aberta-brasileira-o-dtv-play-e-as-suas-potencialidades/

[6] Eutelsat, "Eutelsat Home Page," 2019. line]. Available: https://www.eutelsat.com/en/satellite-communicationservices/broadcasting-solutions.html

[7] M. Takada and M. Saito, "Transmission System for ISDB-T," Proceedings of the IEEE, vol. 94, no. 1, pp. 251-256, jan 2006.

[8] I. Eizmendi, M. Velez, D. Gomez-Barquero, J. Morgade, V. BaenaLecuyer, M. Slimani, and J. Zoellner, "DVB-T2: The second generation of terrestrial digital video broadcasting system," IEEE Transactions on Broadcasting, vol. 60, no. 2, pp. 258-271, 2014.

[9] L. Fay, L. Michael, D. Gomez-Barquero, N. Ammar, and M. W. Caldwell, "An Overview of the ATSC 3.0 Physical Layer Specification," IEEE Transactions on Broadcasting, vol. 62, no. 1, pp. 159-171, mar 2016.

[10] L. Michael and D. Gómez-Barquero, "Bit-Interleaved Coded Modulation (BICM) for ATSC 3.0," IEEE Transactions on Broadcasting, vol. 62, no. 1 , pp. $181-188,2016$.

[11] L. Zhang, W. Li, Y. Wu, X. Wang, S. I. Park, H. M. Kim, J. Y. Lee, P. Angueira, and J. Montalban, "Layered-Division-Multiplexing: Theory and Practice," IEEE Transactions on Broadcasting, vol. 62, no. 1, pp. 216-232, 2016.

[12] P. Klenner, J. S. Baek, N. S. Loghin, D. Gómez-Barquero, and W. S. Ko, "Physical Layer Time Interleaving for the ATSC 3.0 System," IEEE Transactions on Broadcasting, vol. 62, no. 1, pp. 253-262, 2016.

[13] Y. Wang, D. He, L. Ding, W. Zhang, W. Li, Y. Wu, N. Liu, and Y. Wang, "Media Transmission by Cooperation of Cellular Network and Broadcasting Network," IEEE Transactions on Broadcasting, vol. 63, no. 3, pp. 571-576, sep 2017.

[14] G. Bedicks, F. Yamada, F. Sukys, C. E. S. Dantas, L. T. M. Raunheitte, and C. Akamine, "Results of the ISDB-T system tests, as part of digital TV study carried out in Brazil," IEEE Transactions on Broadcasting, vol. 52, no. 1 , pp. $38-44,2006$.

[15] M. Van der Schaar and P. A. Chou, Multimedia over IP and wireless networks: compression, networking, and systems. Elsevier, 2011.

[16] M. Handley, "Why the Internet only just works," BT Technology Journal, vol. 24 , no. 3, pp. 119-129, 2006.

[17] B. Hamzeh, M. Toy, Y. Fu, and J. Martin, "DOCSIS 3.1: Scaling broadband cable to Gigabit speeds," IEEE Communications Magazine, vol. 53 , no. 3 , pp. $108-113,2015$.
[18] I. Cale, A. Salihovic, and M. Ivekovic, "Gigabit passive optical network GPON," Proceedings of the International Conference on Information Technology Interfaces, ITI, pp. 679-684, 2007.

[19] B. Bellalta, "IEEE 802.11ax: High-efficiency WLANS," IEEE Wireless Communications, vol. 23, no. 1, pp. 38-46, 2016.

[20] A. Ghosh, R. Ratasuk, B. Mondal, N. Mangalvedhe, and T. Thomas, "LTE-advanced: Next-generation wireless broadband technology," IEEE Wireless Communications, vol. 17, no. 3, pp. 10-22, 2010.

[21] P. Lescuyer and T. Lucidarme, Evolved Packet Systems (EPS): THE LTE AND SAE EVOLUTION OF $3 G$ UMTS. John Wiley \& Sons Ltd, 2008.

[22] S. S. A. Gilani and M. H. B. Waheed, "QoENGN : A QoE Framework for Video Streaming over Next Generation Mobile Networks," pp. 109$113,2019$.

[23] L. Beloqui Yuste, F. Boronat, M. Montagud, and H. Melvin, "Understanding timelines within MPEG standards," IEEE Communications Surveys and Tutorials, vol. 18, no. 1, pp. 368-400, 2016.

[24] M. L. Wayne, "Netflix, Amazon, and branded television content in subscription video on-demand portals," Media, Culture and Society, vol. 40, no. 5, pp. 725-741, 2018.

[25] T. Spangler, "Cord-cutting alert: Americans watch just $9 \%$ of TV channels available to them," 2014. [Online]. Available: https://variety.com/2014/tv/news/cord-cutting-alertamericans-watch-just-9-of-tv-channels-available-to-them-1201172883/

[26] S. M. Fati, S. Azad, and A.-S. K. Pathan, IPTV Delivery Networks: Next Generation Architectures for Live and Video-on-demand Services. John Wiley \& Sons, 2018.

[27] Y. Liu, Z. Liu, X. Wu, J. Wang, and C. C. Y. Yang, "IPTV system design An ISP's perspective," Proceedings - 2011 International Conference on Cyber-Enabled Distributed Computing and Knowledge Discovery, CyberC 2011, pp. 234-240, 2011.

[28] S. Aslam, "YouTube by the Numbers: Stats, Demographics \& Fun Facts." [Online]. Available: https://www.omnicoreagency.com/youtubestatistics/

[29] N. Erik, S. Ramesh K., and J. Sun, "The Akamai Network: A Platform for High-Performance Internet Applications," ACM SIGOPS Operating Systems Review, 2010

[30] G. M. Lee, C. S. Lee, W. S. Rhee, and J. K. Choi, "Functional Architecture for NGN-Based Personalized IPTV Services," IEEE Transactions on Broadcasting, vol. 55, no. 2, pp. 329-342, 2009.

[31] V. K. Adhikari, Y. Guo, F. Hao, V. Hilt, Z. L. Zhang, M. Varvello, and M. Steiner, "Measurement Study of Netflix, Hulu, and a Tale of Three CDNs," IEEE/ACM Transactions on Networking, vol. 23, no. 6 , pp. 1984-1997, 2015

[32] Sandvine, "The Internet Global Phenomena Report, October 2018," Fremont, CA, USA, Tech. Rep., 2018.

[33] K. J. Ma, R. Bartoš, S. Bhatia, and R. Nair, "Mobile video delivery with HTTP," IEEE Communications Magazine, vol. 49, no. 4, pp. 166-175, 2011.

[34] B. Wang, X. Zhang, G. Wang, H. Zheng, and B. Y. Zhao, "Anatomy of a Personalized Livestreaming System," Proceedings of the 2016 ACM on Internet Measurement Conference - IMC '16, pp. 485-498, 2016.

[35] A. Bentaleb, C. Timmerer, A. C. Begen, and R. Zimmermann, "Bandwidth prediction in low-latency chunked streaming," in Proceedings of the 29th ACM Workshop on Network and Operating Systems Support for Digital Audio and Video - NOSSDAV'19. New York, New York, USA: ACM Press, 2019, pp. 7-13.

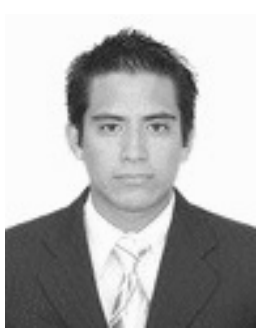

Diego Pajuelo Graduate in Electrical Engineering from the Peruvian University of Applied Sciences (UPC), Lima, Peru in 2012. He is currently working towards his Doctoral degree in Sciences and Telecommunications at Unicamp. His research interests are: HDR Video and audio coding, Image processing, Digital television and Satellite communications. 


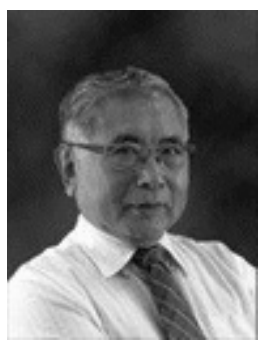

Yuzo Iano is the head and founder of the LCV since 1972. He obtained his BSc (1972), MSc (1974) and $\mathrm{PhD}$ (1986) in Electrical Engineering at Unicamp, SP-Brazil. Research Interests: Digital Signal Processing (images/audio/video), Digital TV, 4G (LTE) and 5G Cellular Networks, Pattern Recognition, Smart Cities, Smart Grid, Internet of Things.

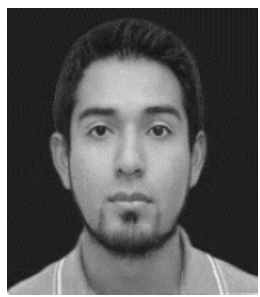

David Minango Graduated in Electronic Engineering at the University Polytechnic Salesian (UPS) Currently, $\mathrm{He}$ is an M.S.c candidate by Department of Communications (DECOM), Faculty of Electrical and Computer Engineering (FEEC) at State University of Campinas (UNICAMP). Professional with engineering experience at Configuration and maintenance of repeater equipment, link antennas, calibration of equipment used in radio frequency in the VHF frequency range for two-way radio equipment. Knowledge in MotoTRB, TCP/IP, WAN Networks. Research Interests: Deep Learning, Machine Learning, Digital Image Processing with Medicals images.

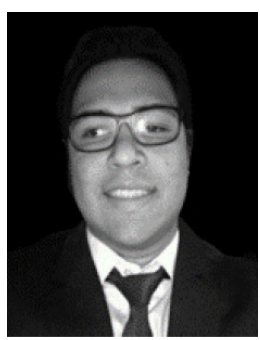

Gabriel Gomes de Oliveira Graduated in Civil Engineering at UNIP University in 2018, studying for a Master's degree at Unicamp, FEEC (Faculty of Electrical and Computer Engineering), DECOM (Communications Department), Laboratory of Visual Communication (LCV) Currently researches and studies the Smart City area.

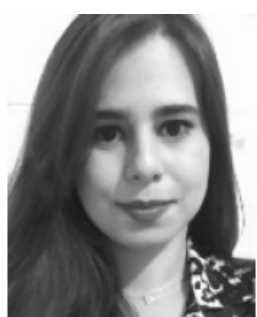

LAB software

Ana Carolina Borges Monteiro Graduated in Biomedicine from University Center Amparense UNIFIA (2015). Currently is a Ph.D. Candidate by Department of Communications (DECOM), Faculty of Electrical and Computer Engineering (FEEC) at State University of Campinas (Unicamp) and a researcher at the Laboratory of Visual Communications (LCV). Also, she is currently the Registration Chair of the Brazilian Symposium on Technology (BTSym). Has expertise in the areas of Clinical Analysis and digital image processing through MAT-

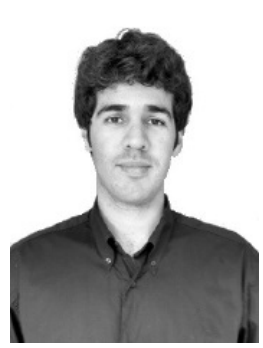

Reinaldo Padilha France Graduated in Computer Engineering (University Regional Center of the Holy Spirit of Pinhal - 2014). Currently is a Ph.D. Candidate by Department of Communications (DECOM), Faculty of Electrical and Computer Engineering (FEEC) at State University of Campinas (Unicamp) and a researcher at the Laboratory of Visual Communications (LCV). Also, He is currently Chair Proceedings of the Brazilian Symposium on Technology (BTSym). Has interest and affinity in the area of technological and scientific research as well as knowledge in programming and development in C / C ++, Java and .NET languages. The main topics of interest are Simulation, Operating Systems, Software Engineering, Wireless and Network, Internet of Things, Broadcasting and Telecommunications Systems. 